\title{
Study of various indexes for Power loss Reduction in Distributed Systems
}

Jaideep ${ }^{* 1}$, Prof. Raman Kamboj ${ }^{2}$

${ }^{*}$ M. Tech, Department of Electrical and Electronics Engineering, Jan Nayak Chaudhary Devi Lal Memorial College of Engineering, Sirsa, Haryana, India

${ }^{2}$ Head of Department, Department of Electrical and Electronics Engineering, Jan Nayak Chaudhary Devi Lal Memorial College of Engineering, Sirsa, Haryana, India

\begin{abstract}
Article Info

Volume 8 Issue 2

Page Number: 380-385

Publication Issue :

March-April-2021

Article History

Accepted : 15 April 2021

Published : 24 April 2021

In swift growing economy, the forceful factors for intensification of Distributed Generation (DG) integration in power distribution system are the expeditious increasing load demands in distribution systems. This research paper work presents load flow program to find out optimal placement and sizing of DG to provide voltage support for the weak distribution system at base case load. Repeated Load Flow (RLF), PSO and SPSO method are used to determine the candidate buses for DG placement in distribution network. Optimal sizing of DG is carried out using RLF with continuous load flow and PSO with random generation of DG sizes.

Keywords : Radial Distribution Network (RDN), Distribution Generation (DG), POWER LOSS , VOLTAGE PROFILE IMPROVEMENT INDEX (VPII).
\end{abstract}

\section{INTRODUCTION}

Voltage Stability is a severe problem in power systems, which steadily reach operating limits imposed by economic and environmental conditions. With the drop in voltage level, the reactive power demand increases. Voltage regulation and reactive power control are essentials in the distribution of electric energy. If the reactive power demand is not met, then it leads to further decline in bus voltage resulting in the cascading effect on neighboring regions. Hence to maintain the voltage profile within permissible limits becomes essential. With the advent of the network and various conditions under which they operate the reactive power and voltage profile management becomes essential so as to ensure the system security and to use the reactive sources in an optimal way. Hence reactive power and its corresponding voltage control has become a critical factor which makes it essential to control. The various methodologies used are the methods branching based on techniques like Sensitivity analysis, Secondary voltage control, evaluation of Voltage stability indices based on network structural characteristics and placement of VAR compensators at suitable locations. Due to the special features of 
distribution networks with radial or weakly meshed structures connected with DG, the operating point of the generators able to optimize the voltage profile in the network may be determined by means of the maximization of the voltages at the load nodes.

$\max V_{i}\left\{f=\sum_{i=1}^{N_{b u x}} V_{i}\right\}$

\section{DISTRIBUTION SYSTEM AND DISTRIBUTED GENERATION}

\section{DISTRIBUTED SYSTEM :}

Distribution system provides a final linkage between the high voltage transmission system and the customers. It is employed with radial structure which is passive in nature has main feeders and lateral distributors. The electrical energy is transported from the production to the consumption, while maintaining an acceptable reliability and voltage quality for all customers through distribution network. Radial Distribution Network (RDN) has high $\mathrm{R} / \mathrm{X}$ ratio, high voltage drops, large power loss due to variation in load. It experiences a sudden voltage collapse at most of the nodes during critical load conditions. Consequently, network power loss increases and voltage profile deteriorates. In this dissertation work, Power Loss Reduction Index (PLRI) and Voltage Profile Improvement Index (VPII) are proposed for all the branches and corresponding nodes respectively. It is observed that node with minimum VPII value is more sensitive and leads to voltage collapse.

Recently, renewable energy sources integration are the best solution to overcome the passiveness of $\mathrm{RDN}$ and also reduce the system losses simultaneously improves the reliability of the system are known as Dispersed or Distribution Generation (DG).

\section{DISTRIBUTED GENERATION:}

The operation and control of distribution system is more complex particularly in the areas where load density is high. Due to uncertainty of system loads on different feeders, which vary infrequently, the modern power system introduces a distribution generation technology in restructured power system. As a result of limited existence of fuel resources, the renewable resources of energy based DG are considered as the alternative solution to existing fuels and well suited for low voltage RDN.

The penetration and operation of Dispersed Generation units in distribution system for the main reason of improving the power system technical performance viz., minimizing network losses, recovers voltage stability, enhance system reliability, improves voltage profile and overall efficiency of distributed electric power. Economical merits include transmission and distribution cost, economize fuel cost and reduce whole sale electricity price from the restructuring point of view. Thus, the dispersion of DG reduces the total amount of electricity cost and maximizes the benefits.

\section{OBJECTIVE FUNCTION}

\section{VOLTAGE PROFILE IMPROVEMENT INDEX (VPII):}

Voltage Profile is a foremost aspect when talks about power distribution system. DG selection can be chosen arbitrary by observing sensitive buses to the voltage collapse and power loss maximization. VPII is another objective function to examine system voltage profile for uniform electric power supply. VPII informs how much voltage profile is affected while implementing DG using various approaches. In this section, VPII is described as ratio of voltage profile with the implementation of DG to voltage profile without DG implementation and given below: 
$V P I I=\frac{V P_{W / D G}}{V P_{W O P D G}}$

\section{POWER LOSS REDUCTION INDEX (PLRI):}

For reduction of system power loss, PRLI objective function has been used to analyze the minimization level of network power loss by implementing DG in distribution network. PLRI can be representing by the equation given below:

$P L R I=\frac{P L_{W / D G}}{P L_{W O / D G}}$

Where, $P L_{W / D G}$ is power loss with DG integration. $P L_{W O / D G}$ is power loss without DG.

\section{MULTI-OBJECTIVE FUNCTION}

For implantation of DG in power distribution system numerous objective functions can be utilized to improve power quality of system. Here, multiobjective function which is combination of both PLRI and VPII is used to minimize the objective function. The multi-objective function can be defined as:

\section{$F=$ minimization $\left(W_{1} f_{1}+W_{2} f_{2}\right)$}

Where, $f_{1}=\operatorname{maximum}(P L R I)$

$\&, f_{2}=$ minimum $\left(\frac{1}{V P I I}\right)$

The $W_{1}$ and $W_{2}$ are the weights having range $0-1$, here equal weightage (0.5) has been taken for the ease of calculation. And, $f_{1}$ and $f_{2}$ are two-single objective functions, where $f_{1}$ is the maximum value of PLRI which has to be minimized and $f_{2}$ is the minimum value of reciprocal of VPII which also has to be minimized. Therefore, the overall objective function ' $F$ ' has to be minimized to obtain optimal solution. The optimal DG size \& placement has been obtained on that candidate bus where overall distribution network power loss is found to be minimized. On the basis of this DG size and location, PLRI and VPII are computed for every branch and each candidate bus.

\section{LOAD FLOW OF RADIAL DISTRIBUTION NETWORKS:}

A feeder brings power from substation to load points/nodes in radial distribution networks (RDN). Single or multiple radial feeders are used in this planning approach. Basically, the RDN total power losses can be minimized by minimizing the branch power flow or transported electrical power from transmission networks (i.e. some percentage of load is locally meeting by local DG). To determine the total power loss of the network or each feeder branch and the maximum voltage deviation are determined by performing load flow. The Forward/Backward Sweep Load Flow technique is used in this case. The impedance of a feeder branch is computed by the specified resistance and reactance of the conductors used in the branch construction. The Forward/Backward Sweep Load Flow method consist two steps (i) backward sweep and (ii) forward sweep. The backward sweep and forward sweep algorithm consists of two basic phases shown in Table 3.1, which are repeated until convergence is achieved.

Table - Backward/ Forward Sweep Method

\begin{tabular}{|c|c|c|}
\hline \multicolumn{3}{|c|}{ Initialize all bus } \\
Voltages & $\begin{array}{c}\text { Backward } \\
\text { Sweep } \\
\text { Algorithm }\end{array}$ & $\begin{array}{c}\text { Sum currents or power } \\
\text { flows (Possibly update } \\
\text { voltages) }\end{array}$ \\
\hline 2. & $\begin{array}{c}\text { Forward } \\
\text { Sweep } \\
\text { Algorithm }\end{array}$ & $\begin{array}{c}\text { Calculate voltage drop } \\
\text { (Possibly update } \\
\text { currents/power flows) }\end{array}$ \\
\hline \multicolumn{2}{|c|}{ Repeat steps 1 and 2 until convergence is } \\
\end{tabular}

Backward Sweep: In this step, the load current of each node of a distribution network having $\mathrm{N}$ number of nodes is determined as: 
$\bar{l}(m)=$
$\frac{p_{l}(m)-\rho_{l}(m)}{\vec{v}}$

$1,2,3, \ldots \ldots \ldots m]$

Where, $P_{L}(m)$ and $Q_{L}(m)$ represent the active and reactive power demand at node $m$ and the over bar notation $(\bar{x})$ indicates the phasor quantities, such as $\overline{I_{L}}, \overline{V^{*}}$. Then, the current in each branch of the network is computed as:

$\bar{I}(m n)=\overline{I_{L}}(n)+\sum_{m \in \Gamma} \overline{I_{L}}(m)$

where, the set $\Gamma$ consists of all nodes which are located beyond the node $\mathrm{n}$ [9].

Forward sweep: This step is used after the backward sweep so as to determine the voltage at each node of a distribution network as follows:

$\bar{V}(n)=\bar{V}(m)-\bar{I}(m n) Z(m n)$

where, nodes $\mathrm{n}$ and $\mathrm{m}$ represent the receiving and sending end nodes, respectively for the branch $\mathrm{mn}$ and $\mathrm{Z}(\mathrm{mn})$ is the impedance of the branch.

In this work the estimation methodology utilized within the forward/backward load flow is based on (i) equivalent current injections (ECI), (ii) the nodeinjection to branch current matrix (BIBC) and (ii) the branch-current to node-voltage matrix (BCBV). In this area, the advancement methodology will be depicted in subtle element. Load flow for distribution networks under balanced operating condition with constant power load model can be under remained through the accompanying focuses:

\section{EQUIVALENT CURRENT INJECTION:}

The technique is based on the equivalent current injection of a node in distribution networks, the equivalent-current-injection model is more practical. For any node of distribution networks, the complex load $S_{i}$ is expressed as:
$S_{i}=$
$P_{i} \dagger$
$i Q_{i}$
$1_{1} \ldots \ldots \ldots N_{B}$

Now, the equivalent current injection is expressed as: $I_{i}=I_{i}^{r}\left(V_{i}\right)+j I_{i}^{i}\left(V_{i}\right)=$

$\left(\frac{P_{i}+j Q_{i}}{V_{i}}\right)^{*}$ $i=$ $1, \ldots \ldots N_{B}$

For the load flow solution equivalent current injection (ECI) at the $\mathrm{k}$-th iteration at $\mathrm{i}$-th node is computed as:

$I_{i}^{k}=I_{i}^{r}\left(V_{i}^{k}\right)+j I_{i}^{i}\left(V_{i}^{k}\right)=\left(\frac{P_{i}+j Q_{i}}{V_{i}}\right)^{*}$

\section{FORMATION OF BIBC MATRIX:}

The power injections at every node might be transformed into the equivalent current injections using the eq. (3.12) and applying Kirchhoff's Current Law (KCL) at each and every node a set of comparisons could be composed. Now each and every branch currents of the network can be shaped as a function of the equivalent current injections (ECI) [10- 11] [12]. As shown in Figure 3.1, the branch currents $I_{B 1}, I_{B 2}, I_{B 3}, I_{B 4}$, and $I_{B 5}$ can be expressed as:

$I_{B 1}=I_{2}+I_{3}+I_{4}+I_{5}+I_{6}$

(3.12)

$I_{B 2}=I_{3}+I_{4}+I_{5}+I_{6}$

(3.13)

$I_{B 3}=I_{4}+I_{5}$

(3.14)

$I_{B 4}=I_{5}$

(3.15)

$I_{B 5}=I_{6}$ 


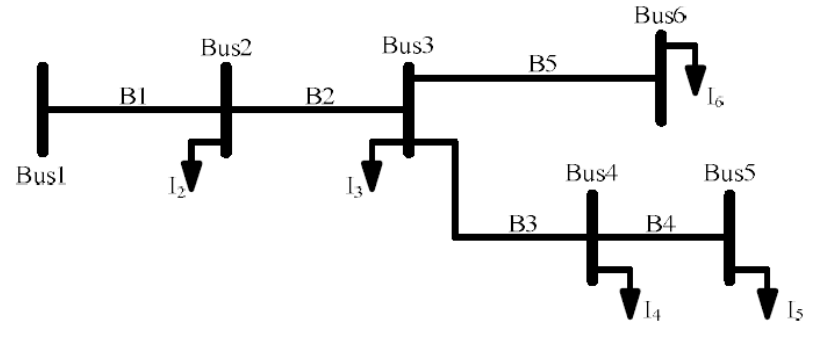

Figure 3.1. Simple Distribution System

From the above equations () the BIBC matrix can be written as:

$\left[\begin{array}{l}I_{B 1} \\ I_{B 2} \\ I_{B 3} \\ I_{B 4} \\ I_{B 5}\end{array}\right]=\left[\begin{array}{lllll}1 & 1 & 1 & 1 & 1 \\ 0 & 1 & 1 & 1 & 1 \\ 0 & 0 & 1 & 1 & 0 \\ 0 & 0 & 0 & 1 & 0 \\ 0 & 0 & 0 & 0 & 1\end{array}\right]\left[\begin{array}{l}I_{1} \\ I_{2} \\ I_{3} \\ I_{4} \\ I_{5}\end{array}\right]$

(3.17)

The general form as of eq. (3.17) can be expressed as:

$\left[I_{B}\right]=[B I B C]\left[I_{n}\right]$

The detailing of BIBC matrix for distribution networks demonstrated in Figure 3.1 is given in eq. (3.18). For general network, the BIBC matrix might be shaped through the accompanying steps and the example is done by the help of Figure 3.1:

Step 1: Make an initial null BIBC matrix with a dimension of $(m \times(n-1))$, Where $m$ and

$\mathrm{n}$ are the number of branches and nodes available in the network.

Step 2: Initially set $\mathrm{i}=1$ and read the $\mathrm{I}_{B i}(\mathrm{i}=1,2,3 \ldots \mathrm{m})$ branch data (i.e., sending end and receiving

end node) from line data matrix. If a line section IBi is located between node ' $x$ ' and node

' $y$ '. Check that $I_{B i}$ branch section of the network belongs to the first node of the network

or not. If it is, then make the (y-1, y-1)-th bit of BIBC matrix by ' +1 '. Increment ' $i$ ' by

one or go to the step-3.

Step 3: If the IBi branch section does not belong to the first node of the network in the step- 2 .

Then, '( $\mathrm{x}-1)$ '-th node of BIIBC matrix to the column segment of ' $(y-1)$ '-th node and fill

$(y-1, y-1)$-th bit of the BIBC matrix by ' +1 '. Increment ' $\mathrm{i}$ ' by one and go to the step- 2 .
This is explained in figure 3.2:

Step 4: Repeat step\#2 and step\#3 until all the branches of the network included in to the BIBC matrix.

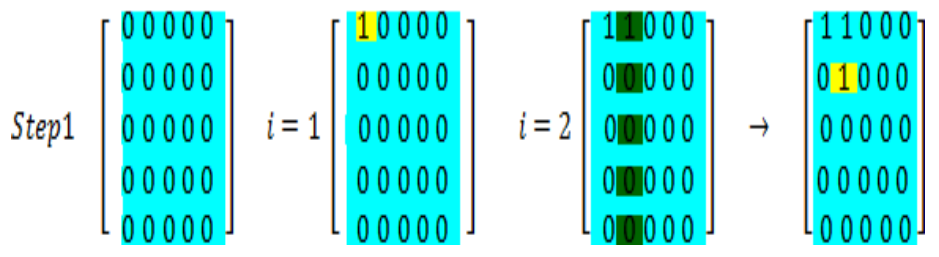

In step 2 Copy $1^{\text {st }}$ column $\quad$ In step 3

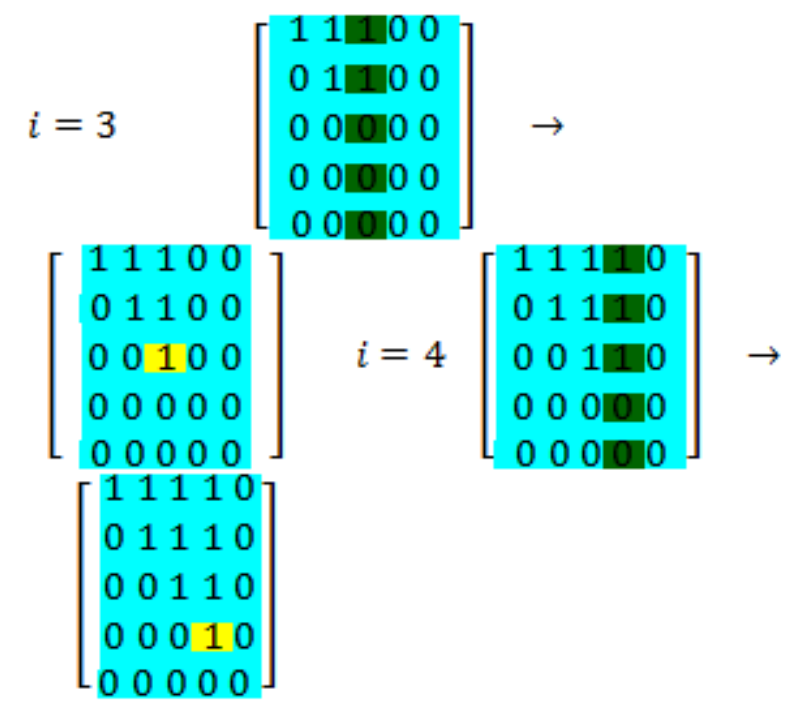

Copy $2^{\text {nd }}$ column In step 3 Copy $3^{\text {rd }}$ column

In step 3

$i=4$

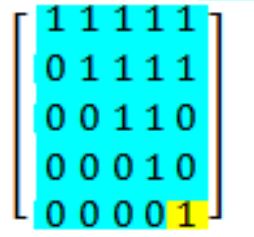

Copied row Updated row

Copy $2^{\text {nd }}$ column In step 3

\begin{tabular}{|c|c|c|}
\hline Branch No. & Sending End & Receiving End \\
\hline 1 & 1 & 2 \\
\hline 2 & 2 & 3 \\
\hline 3 & 3 & 4 \\
\hline 4 & 4 & 5 \\
\hline 5 & 3 & 6 \\
\hline
\end{tabular}


Figure The formation of BIBC matrix, the example is done by the help of Figure 3.1.

\section{CONCLUSION}

In this research work, voltage profile improvement and power losses minimization have been done by placing DG in an optimal location in RDN using distinct techniques. The merits of placing DG for improving voltage profile and reducing power loss is analyzed in a detailed manner. Several test case studies are carried out on IEEE-12, 33 and 69 bus radial distribution networks. The DG unit placement problem has been optimized by implementing distinct techniques using MATLAB® 13.0 found to be nurturing. In IEEE-12 bus system, power loss reduction percentages are $43.363 \%, 43.438 \%$ and $49.587 \%$ for RLF, PSO, and SPSO respectively.

\section{REFERENCES}

[1]. Rajkumar Viral, and D. K. Khatod, "Optimal Planning of Distributed Generation Systems in Distribution System: A Review", Renewable and Sustainable Energy Reviews 16, pp. 51465165, 2012.

[2]. P. Ajay-D-Vimal Raj, S. Senthilkumar, J. Raja, S. Ravichandran, and T. G. Palanivelu, "Optimization of distributed generation capacity for line loss reduction and voltage profile improvement using PSO," ET AL. /Elektrika, vol. 10, no. 2, pp. 41-48, 2008.

[3]. R. Srinivasa Rao, K. Ravindra, K. Satish, and S. V. L. Narasimham, "Power loss minimization in distribution system using network reconfiguration in the presence of distribution generation," IEEE Trans. on Power Systems, vol. 28, no. 1, pp. 317-325, Feb. 2013.

[4]. SooHyoung Lee, and Jung-Wook Park, "Optimal placement and sizing of multiple DGs in a practical distribution system by considering power loss," IEEE Trans. on Industry Applications, vol. 49, no. 5, Sep. /Oct. 2013.

[5]. C.L.T. Borges and D.M. Falcao, "Optimal Distributed Generation Allocation for Reliability, Losses and Voltage Improvement", International journal of power and energy systems, vol.28.no.6, pp.413-420, July 2006.

[6]. G. Celli, E. Ghiani, S. Mocci, F. Pilo, "A MultiObjective Evolutionary Algorithm for the Sizing and Siting of Distributed Generation", IEEE Trans. Power Systems, vol. 20, no. 2, pp. 750-757, 2005.

[7]. G. Carpinelli, G. Celli, S. Mocci, F. Pilo, A. Russo, "Optimisation of Embedded Generation Sizing and Siting by using a Double Trade-Off Method," IEE Proc.-Gener. Transm. Distrib., vol. 152, no. 4, pp. 503-513, 2005.

\section{Cite this article as :}

Jaideep, Prof. Raman Kamboj, "Study of various indexes for Power loss Reduction in Distributed Systems", International Journal of Scientific Research in Science, Engineering and Technology (IJSRSET), Online ISSN : 2394-4099, Print ISSN : 2395-1990, Volume 8 Issue 2, pp. 380-385, March-April 2021. Available at

doi : https://doi.org/10.32628/IJSRSET218288

Journal URL : https://ijsrset.com/IJSRSET218288 